\title{
Alcoolismo materno e as implicaÇões no Cuidado da CRIANÇA: estudo QUALITATIVO
}

\author{
Ana Cristina Pereira de Jesus Costa ${ }^{1}$ \\ Priscila Morgana da Silva ${ }^{2}$ \\ Priscila Coimbra Rocha ${ }^{3}$ \\ Márcio Flávio Moura de Araújo ${ }^{4}$ \\ Thiago Moura de Araújo ${ }^{4}$ \\ Neiva Francenely Cunha Vieira
}

\begin{abstract}
Objetivou-se, nesta pesquisa qualitativa, avaliar como é realizado o cuidado da criança por mães alcoolistas. Realizaram-se entrevistas em profundidade com 10 mães alcolistas, no período de dezembro de 2011 a janeiro de 2012, em dois serviços de atenção em saúde mental do município de Imperatriz, MA. Os dados foram analisados confome a técnica de análise de conteúdo proposta por Bardin. Observou-se que o cuidado realizado pelas mães em dependência de álcool predispõe a criança a vulnerabilidades à sua saúde, pois afeta a capacidade cognitiva e as habilidades maternas para o cuidado infantil. $\mathrm{O}$ estudo possibilitou observar a importância dessa proposta de investigação realizada por enfermeiros, para a elaboração de estratégias de enfrentamento do alcoolismo e de trocas de experiência sobre o cuidado adequado da criança junto às mães alcoolistas.
\end{abstract}

Descritores: Alcoolismo; Mães; Criança; Enfermagem.

\footnotetext{
${ }^{1}$ Doutoranda, Universidade Federal do Ceará, Fortaleza, CE, Brasil. Professor Assistente, Universidade Federal do Maranhão, Imperatriz, MA, Brasil.

2 Enfermeira, Hospital Geral e Maternidade de Pedreiras, Pedreiras, MA, Brasil.

3 MSc, Professor Assistente, Universidade Federal da Bahia, Salvador, BA, Brasil.

${ }^{4} \mathrm{PhD}$, Professor Adjunto, Universidade da Integração Internacional da Lusofonia Afro-Brasileira, Redenção, CE, Brasil.

${ }^{5}$ PhD, Professor Titular, Universidade Federal do Ceará, Fortaleza, CE, Brasil.
} 


\section{Maternal ALCOHOLISM AND CHILdCARE IMPLICATIONS: A QUALITATIVE STUDY}

The aim of this qualitative research was to evaluate how alcoholic mother care for their children. In-depth interviews were conducted with 10 alcoholic mothers between December 2011 and January 2012, in two mental health care services in the municipality of Imperatriz, MA. The data were analyzed according to Bardim's content analysis technique. The care provided by alcohol dependent mothers predisposes the children to vulnerable health, as the mother's cognitive capacities and childcare skills are affected. The study shows the importance of this investigation by nurses in order to create strategies to deal with alcoholism and to exchange experiences on appropriate childcare together with alcoholic mothers.

Descriptors: Alcoholism; Mothers; Child; Nursing.

\section{Alcoholismo materno y las implicaCiones en el CUIDAdo Del niño: ESTUDIO CUALITATIVO}

Se objetiva, en este estudio cualitativo, validar como es realizado el cuidado del niño por madres alcohólicas. Se realizaron entrevistas en profundidad con 10 madres alcohólicas, en el período de diciembre de 2011 a enero de 2012, en dos servicios de atención de salud mental del municipio de Imperatriz, MA. Los datos fueron analizados conforme a la técnica de análisis de contenido propuesta por Bardin. Se observó que el cuidado realizado por las madres con dependencia del alcohol predispone al niño a ser vulnerable en materia de salud, pues afecta a la capacidad cognitiva y a las habilidades maternas para el cuidado infantil. El estudio posibilitó observar la importancia de esa propuesta de investigación realizada por enfermeros, para la elaboración de estrategias de enfrentamiento del alcoholismo y de intercambio de experiencias sobre el cuidado adecuado del niño junto a las madres alcohólicas.

Descriptores: Alcoholismo; Madres; Niño; Enfermería.

\section{Introdução}

O alcoolismo corresponde a um importante problema de saúde pública, uma vez que compromete o cotidiano social e familiar e, portanto, implica a necessidade de se obter mais conhecimento para o seu enfrentamento ${ }^{(1)}$. Durante muito tempo, o alcoolismo esteve relacionado à população masculina como forma de vinculação social. No entanto, nos últimos anos observa-se, em todo o mundo, crescente aumento do alcoolismo feminino ${ }^{(2)}$.
O panorama do alcoolismo entre as mulheres dobrou a partir do ano 2001 e alcançou a proporção de uma mulher para cada três homens alcoolistas, o que demonstra a suscetibilidade cada vez maior das mulheres para o uso de álcool. Dados do II Levantamento Domiciliar sobre o Uso de Drogas Psicotrópicas no Brasil, envolvendo as 108 maiores cidades do país, apontam que $6,9 \%$ das mulheres brasileiras são dependentes de álcool. Além disso, a recorrência anual de mulheres aos 47 Centros de Atenção Psicossocial Álcool e outras Drogas (CAPS Ad) 
no Brasil, para tratamento do alcoolismo, quantificam um valor estimado de 3.000 mulheres nessa situação ${ }^{(3)}$.

Estudos que abordaram o consumo de álcool entre as mulheres consideram que o alcoolismo feminino é capaz de afetar o processo de expressão e de relacionamentos afetivos, podendo comprometer substancialmente a interação mãe/ filho, uma vez que, para o cuidado da criança, é fundamental a condição saudável da mãe $e^{(4-5)}$. Nesse sentido, o cuidado materno dispensado por mães alcoolistas pode atuar como preditor negativo para a futura saúde dessas crianças ${ }^{(5-6)}$.

O cuidado materno representa, pois, uma ação integral capaz de proporcionar a disseminação do carinho e do amor entre os componentes da família. O cuidado é uma arte, sendo necessário utilizar-se de critérios básicos como afeto, sensibilidade e habilidade. Para que haja efetivação do cuidado da criança, é essencial que a mãe apresente suas funções cognitivas preservadas e seja capaz de oferecer condições adequadas de cuidado, bem como ser acolhedora às necessidades do filho, gerando ambiente favorável ao desenvolvimento hígido da criança. Quando o cuidado não acontece conforme o esperado, a criança pode tornar-se deprimida, psicologicamente incapaz de se relacionar, apática ou até mesmo evoluir ao óbito ${ }^{(7)}$.

Investigações dessa natureza ressaltam o papel do profissional de saúde na prevenção e detecção dos problemas relacionados aos cuidados realizados por mães alcoolistas, pois retrata um processo que pode ser difícil e inviável à sobrevida ou ao desenvolvimento saudável da criança, por comprometer o cotidiano social e familiar e afetar a capacidade da mãe na realização de cuidados à criança.

Este estudo mostra-se relevante devido ao impacto causado pelo alcoolismo na família, e entendendo que a sua presença repercute na saúde de todos os membros, o cuidado executado por mães em condição de dependência alcoólica representa fator determinante para o desenvolvimento saudável da criança. Justifica-se, também, por contribuir, diante das suas possíveis utilidades, para a aplicação à realidade das práticas dos serviços de saúde brasileiros e, com isso, contribuir para o direcionamento do corpo de conhecimentos em enfermagem sobre o alcoolismo materno. Em face do exposto, este estudo teve como objetivo avaliar como é realizado o cuidado da criança por mães alcoolistas.

\section{Método}

Trata-se de estudo descritivo, com abordagem qualitativa, realizado no Centro de Atenção Psicossocial Álcool e outras Drogas (CAPS Ad) e na Comunidade Terapêutica Casa de Rute, no município de Imperatriz, Maranhão, Brasil.

Os locais escolhidos para a realização deste estudo são serviços de saúde para o tratamento e a prevenção do uso/abuso de álcool e outras drogas. As referidas instituições apresentam aspectos distintos de funcionamento: a Comunidade Terapêutica Casa de Rute caracteriza-se por ser uma instituição filantrópica que oferece abrigo e assistência a pacientes com dependência química, tendo acolhido, em média, doze pessoas durante o período da pesquisa. O CAPS Ad possui equipe de saúde multiprofissional semanal, além de condições estruturais e funcionais para arteterapia, musicoterapia, área didática, área para lazer e grupos de reunião entre pacientes e familiares.

As participantes do estudo foram 10 mães alcoolistas que concordaram em participar do estudo, assinando previamente o Termo de Consentimento Livre e Esclarecido (TCLE). Dentre os critérios de elegibilidade para a pesquisa, estão: ser mãe alcoolista e estar em tratamento nos serviços de tratamento e prevenção ao uso de drogas e álcool.

Entrevistas em profundidade foram realizadas em espaços terapêuticos dos respectivos serviços, após consentimento das participantes. Cada mãe foi entrevistada duas vezes, ademais, esses encontros foram gravados com auxílio de um aparelho MP4 e, posteriormente, transcritas e analisadas. A duração média de cada entrevista oscilou entre 10 e 15 minutos. Para nortear esses momentos, foram pontuados os seguintes tópicos semiestruturados, a saber: alimentação, higiene, prevenção de doenças e educação no âmbito escolar e familiar.

Os dados foram analisados sob a perspectiva da análise de conteúdo de Bardin $^{(8)}$, constituída por três etapas: pré-análise; exploração do material e tratamento dos resultados obtidos. 
A pesquisa foi aprovada pelo Comitê de Ética em Pesquisa do Hospital Universitário da Universidade Federal do Maranhão, sob Parecer 223/11, em 29 de agosto de 2011, em anteparo à Resolução 196/96, do Conselho Nacional de Saúde ${ }^{(9)}$.

\section{Resultados e Discussão}

Caracterização das participantes da pesquisa

As participantes foram 10 mães alcoolistas, com faixas etárias diferenciadas, média da idade mínima de 22 anos e idade máxima de 46 anos. No que concerne à atividade laboral, quatro estavam desempregadas, duas eram domésticas, uma pensionista e três possuíam ocupações informais. Dentre as participantes, três eram casadas e as demais solteiras, e, em média, tinham de dois a seis filhos. A faixa etária dos filhos variou de três a dez anos, três desses possuíam idade entre 3 e 5 anos e os demais estavam inclusos na faixa etária de 5 a 10 anos. Vale salientar que todas as participantes do estudo estavam em fase de abstinência.

No presente estudo observou-se que apenas duas das entrevistadas utilizavam os serviços do CAPS Ad, em contrapartida, oito frequentavam regularmente a instituição filantrópica Casa de Rute, evidenciando uma lacuna no atendimento prestado às mães alcoolistas pelo CAPS Ad.

O CAPS Ad possui relevante papel no acompanhamento de alcoolistas, por se caracterizar como instituição de caráter multiprofissional, baseado num modelo de atenção integral à saúde, que preconiza a mudança da ideia de doentes para a de cidadãos, promovendo reinserção social e intersetorialidade das ações, com adoção da política de redução de danos e outros princípios para atenção integral, justa e equânime ${ }^{(10)}$. Aponta, pois, a necessidade de reformulação na assistência ofertada a esse público em nível ambulatorial e domiciliar pelo Sistema Único de Saúde, demonstrando a necessidade de reelaboração e renovação de políticas públicas direcionadas a essas usuárias.

Alcoolismo materno e o processo de cuidar

Os aspectos do cuidado da criança analisados, que podem sofrer interferências diante da presença do alcoolismo materno, são: alimentação e higiene da criança, educação dos filhos, amor/atenção dispensados aos filhos, caráter psicológico e emocional da criança, dificuldades na provisão de cuidados maternos e riscos à saúde da criança.

A oferta de fontes alimentares balanceadas, em tempo hábil e contínuo, na presença da figura materna, representa fator essencial para a manutenção do crescimento e desenvolvimento hígido da criança. Entretanto, a oferta insuficiente de alimentos pode inferir em debilidade física e imunológica, e acarretar adoecimento infantil. Nessa perspectiva, a alimentação representa uma dimensão privilegiada do cuidado, devendo ser conivente com a oferta de alimentos, adequadamente, compatíveis com a faixa etária e necessidades da criança, evitando, assim, o adoecimento $^{(11)}$.

Em virtude da sua condição de dependência alcoólica, as mães alcoolistas podem deteriorar vínculos e menosprezar a relevância de cuidados com a alimentação dos filhos, conforme se pode vislumbrar nos depoimentos a seguir.

[...] eu não estava me importando com a comida deles [...] Eu queria saber era de tomar cachaça (Turquesa).

Meus filhos pequenos muitas vezes dormiram sem jantar [...] (Rubi).

Os depoimentos demonstram as repercussões avassaladoras do alcoolismo no âmbito familiar, sendo capaz de afetar o elo e interferir diretamente na responsabilidade em prover a alimentação e, consequentemente, implicando diretamente a saúde da criança.

No que diz respeito à ausência de cuidados com a higiene corporal da criança, evidencia-se o descuido, de acordo com o depoimento de Rubi.

[...] eu já não conseguia ficar sem beber álcool, e nesse intervalo eu fui esquecendo o meu lado de mãe, porque meus filhos dormiam sem tomar banho [...] (Rubi).

O depoimento explicita, além do descuido, sentimentos de arrependimento/culpa maternos diante do alcoolismo, evidenciando disfunção do papel materno diante da atenção à higiene da criança, fator capaz de ocasionar a aquisição de diversas doenças provenientes da sujidade e, sobretudo, ausência de higidez e bem-estar físico da prole.

Corroborando tal afirmação, autores apontam a importância da higiene da criança como 
necessidade básica de manutenção da saúde e exercício da construção de hábitos saudáveis, na perspectiva da valoração do cuidado. Nesse contexto, ressaltam, ainda, que as ações de atenção à higiene da criança representam um domínio dos cuidados necessários para o desenvolvimento saudável das crianças ${ }^{(9,12)}$.

Com o avanço da dependência alcoólica e ausência de tratamento específico, há exacerbação dos sintomas e aumento do egocentrismo da mãe alcoolista, que menospreza, também, o processo educacional dos filhos, conforme é enfatizado por Pérola em seu depoimento.

As crianças não tiveram uma boa educação diante da mãe que eu fui [...] O filho não vai mais à praia, a um clube, nem na casa de parentes, porque a mãe está bêbada (Pérola).

Percebe-se, no depoimento, que há perda progressiva do vínculo entre o binômio mãe/ filho. A ausência de orientações corretas pode comprometer, significativamente, a índole e o lado psicológico da criança, podendo acarretar alterações comportamentais irreversíveis, inclusive quando atrelada à ausência ou insuficiência de entretenimento/lazer familiar.

Vários estudos nacionais e internacionais têm apresentado a influência parental no ambiente familiar como mecanismo colaborador à vulnerabilidade dos filhos para a dependência alcoólica $^{(12-13)}$.

Não obstante, nos casos de alterações de conduta, vale salientar que os filhos são verdadeiramente um reflexo dos pais, logo, podem internalizar reações agressivas e condutas refutáveis, integrando sua personalidade e ocasionando uma espécie de "efeito cascata" para com as futuras gerações. Nesse sentido, os pais são as principais referências para as crianças, os primeiros educadores e, portanto, representantes das formas corretas de conduta a serem seguidas ${ }^{(13)}$.

Comportamentos agressivos ou irracionais, como consequência do efeito do álcool, também são evidenciados, conforme se pode verificar nos depoimentos de Pérola e Safira, respectivamente.

Eu levava para o bar, colocava numa cadeira, o baby conforto (Pérola).

Eu levava minha filha para os bares. Ela dormia no meu colo e acordava quando eu estava derramando a bebida nela (Safira).

Percebe-se um disparate no processo educacional dos filhos, constituindo comprometimento da segurança da criança e influência direta para a adesão ao alcoolismo, visto que a criança pode internalizar as condutas da mãe alcoolista e isso pode influenciar decisivamente o comportamento posterior da prole.

As atitudes implícitas e demonstrações públicas de afeto são fundamentais, pois suscitam na criança sensações de aconchego, proteção, serenidade, alegria, gratificação e bem-estar imensuráveis, bem como reações de reciprocidade. Nessa perspectiva, essa forma de cuidado é essencial para o fortalecimento do vínculo afetivo e amistoso entre a mãe e o filho. Entretanto, o alcoolismo gera episódios recorrentes de agressão mútua, minimizando a provisão de afeto da mãe aos filhos e despendendo menos tempo para os cuidados dos quais eles necessitam ${ }^{(13-14)}$.

A dependência alcoólica gera um egocentrismo tamanho, de tal modo que os esforços de manutenção do vínculo entre a díade mãe/álcool são suficientes para a exacerbação do "bem-estar" individual da mãe, em detrimento das necessidades afetivas da criança, conforme é demonstrado depoimentos abaixo.

Dava atenção nenhuma. Não sabia nem se era filho (Turquesa).

Nunca dei atenção e carinho. A pessoa que bebe não sabe amar criança (Safira).

Tais afirmações evidenciam o abismo que há na relação entre a mãe alcoolista e os seus filhos, fator que contribui para tornar a criança vulnerável a desordens generalizadas, especialmente de conduta ou psicopatológicas.

No que tange aos efeitos psicoemocionais que acometem a criança, filha de mãe alcoolista, as adversidades podem repercutir de forma intensa, conforme é ressaltado adiante.

[...] a criança absorve o nervosismo da mãe. Do jeito que eu tratava, eles vão passar a tratar outras pessoas [...] O futuro da criança é ansiedade, insegurança, é o que os meus filhos têm (Pérola).

Diante dos depoimentos supracitados, percebe-se que o processo de maturidade emocional e desenvolvimento psicossocial da criança, filho(a) de mãe alcoolista, sofrem intenso curso de deterioração, na maioria das vezes irreversível e responsável por comportamentos que podem se manifestar em forma de agressividade, dificuldades de aprendizagem e, principalmente, dificuldade de se relacionar com outras pessoas ${ }^{(14)}$. 
Na presença do alcoolismo e de algumas comorbidades associadas, tais como a depressão materna, as atribuições maternas no cuidado da criança e de promoção do seu crescimento e desenvolvimento saudáveis estão comprometidas. De acordo com os relatos, as dificuldades na provisão de cuidados maternos podem ser traduzidas, como mostrado a seguir.

O álcool me atrapalhou porque eu não sabia ser mãe, não me deixava ser mãe (Pérola).

Eu me sentia fracassada, incapaz de ser mãe [...] Eu queria voltar a ser mãe de verdade, esposa de verdade, mulher de verdade (Rubi).

As dificuldades percebidas no cuidado da criança estão intrinsecamente relacionadas à condição de dependência alcoólica que se sobrepõe às prioridades vitais do ser humano. Nessa perspectiva, o alcoolismo materno deteriora a responsabilidade, traduzindo-se na incapacidade de trabalhar, de cuidar de si mesma e, principalmente, de ser mãe $e^{(15)}$.

Há casos em que se evidencia perda da confiança dos filhos em relação à mãe, conforme se pode observar na fala abaixo.

Quando eu passo da hora de chegar, meus filhos ligam para perguntar onde estou. Eu digo que estou no serviço, não acreditam [...] (Pérola).

Percebe-se que, com o tempo, a criança subestima a mãe e a sua capacidade de ser mãe e cuidadora responsável e começa a denominá-la fraca, leviana e incapaz, perdendo a confiança na mesma.

O apoio familiar pode amenizar essa problemática, subsidiando a provisão de cuidados $^{(15-16)}$. De fato, a família deve estar predisposta e integrada nessa ação que requer bastante esforço e compreensão por parte de todos os envolvidos.

Determinadas condutas de mães alcoolistas deixam a criança vulnerável à morbidade severa ou até mesmo à mortalidade. As dificuldades em abandonar a bebida alcoólica para adquirir serenidade para a adequada provisão de cuidados aos filhos, de tal modo que garanta a saúde da criança, foram percebidas no presente estudo, em que, dentre as dez participantes, sete delas referiram estar em consumo abusivo de bebida alcoólica desde a fase da adolescência, perpetuando o consumo, inclusive, durante a gestação e nos períodos de amamentação, conforme referido no depoimento a seguir.
Quando eu comecei a ingerir bebida alcoólica eu estava grávida [...] (Rubi).

A ingestão de álcool na gravidez predispõe ao óbito materno e infantil, quer seja por aborto prematuro, acidentes ou incidentes de múltiplas causas, ou até mesmo por aquisição da síndrome alcoólica fetal, dentre outros fatores que podem afetar, de forma impactante, a saúde e sobrevida do concepto, ocasionando sequelas, como o retardo mental, alterações faciais típicas, dificuldades de crescimento e desenvolvimento e da cognição ${ }^{(16)}$.

A amamentação é contraindicada em uso de bebidas alcoólicas, uma vez que o álcool liberado no leite materno funciona como componente bastante prejudicial ao desenvolvimento psicomotor da criança, além de alterar o sabor do leite, minimizando os episódios de sucção e o processo de nutrição infantil. A bebida alcoólica transfere-se para o leite materno a partir do consumo de $1 \mathrm{~g} / \mathrm{kg} /$ dia. Dessa forma, a ingestão prolongada de álcool na gestação/lactação pode ser capaz de provocar alterações e efeitos no feto e no recém-nascido, podendo acarretar prejuízos na capacidade de aprendizagem, memória e aumento da mortalidade infantil ${ }^{(17-18)}$.

Ainda, dentre as entrevistadas, quatro admitiram espancar o filho quando sob os efeitos da bebida alcoólica, capaz de ocasionar repercussões físicas e psicológicas desastrosas. Essa situação pode ser constatada pelos depoimentos adiante.

Embriagada eu batia neles sem motivo (Turquesa).

Eu era muito agressiva com meus filhos (Esmeralda).

Condutas maternas agressivas por efeito da bebida alcoólica, demonstrativas de ausência do vínculo materno infantil, representam fatores graves de risco à saúde da criança, sendo capaz de afetar essencialmente a relação mãe/filho, conforme é evidenciado abaixo.

Eles percebiam quando eu estava bêbada. [...] O meu filho mais velho deixou até de me pedir a bênção (Turquesa).

O alcoolismo materno distancia mãe/filho, afetando a relação interpessoal nesse sentido. Nesse aspecto, as intervenções de enfermagem no âmbito familiar representam uma ação crucial a se considerar. $\mathrm{O}$ enfermeiro pode intervir sobre diversos fatores problemáticos que compõem o universo familiar e que afetam a saúde dos membros. Dessa forma, adota a família como 
unidade de cuidado, considerando a identidade da mesma, bem como os seus dilemas peculiares; os comportamentos interpessoais na esfera familiar; avaliando o funcionamento diário da família, bem como a presença de fatores de risco à saúde dos membros; realizando visitas periódicas com a equipe multiprofissional e subsídio do NASF (Núcleo de Apoio à Saúde da Família) e propondo condições precisas de cuidado, assistência e tratamento à mãe alcoolista e aos demais integrantes do núcleo familiar, especialmente diante da presença do alcoolismo materno ${ }^{(18)}$.

Consoante os dados apresentados, observa-se que as participantes possuíam consciência da gravidade do seu hábito etílico em relação ao cuidado de seus filhos. Dessa maneira, é razoável admitir que isso acentua o sofrimento dessas mulheres, sua sensação de culpabilidade e marginalização social. Assim, é imperativo que os profissionais de saúde escutem reflexivamente essas mulheres, executem ações de acolhimento e de tratamento equânime e imparcial.

\section{Conclusão}

A partir dos resultados identificados, elaborou-se a definição de que o alcoolismo materno afeta o processo de cuidado dispensado às crianças, comprometendo o desenvolvimento emocional e psicossocial de vida, gerando impacto negativo à saúde da criança.

A conclusão deste estudo remete aos efeitos e repercussões do alcoolismo materno no âmbito familiar diante do cuidado com a criança, levando à observação de que o alcoolismo é uma doença altamente complexa, deletéria e de ação generalizada, que provoca adoecimento não apenas da criança, como de todos os integrantes da família, desestruturando primordialmente sua base de funcionamento - a figura materna, predispondo os filhos aos riscos de morbimortalidade biológica e mental, comprometendo a maturidade emocional da criança e desequilibrando a integridade do lar.

Historicamente, a mulher é culpabilizada pelos erros nos cuidados e educação dos filhos, gerenciamento do lar e da família em geral. Contudo, essa visão é equivocada e preconceituosa, pois alguns fatores estão relacionados a esse quadro nas mães alcoolistas, a saber: rede social de apoio deficiente, vulnerabilidade socioeconômica e cognitiva e, sobretudo, falta de afeto e compreensão com o estado vigente.

$\mathrm{O}$ apoio familiar integral bem como o aumento dos laços afetivos, amparados por redes sociais de acompanhamento são fundamentais, pois facilitam o restabelecimento materno, suscitando a relevância de adesão ao tratamento e aprimorando a qualidade de vida dos integrantes do núcleo familiar.

\section{Referências}

1. Santos ECV, Martin D. Cuidadoras de pacientes alcoolistas no município de Santos, SP, Brasil. Rev Bras Enferm. 2009;62:194-9.

2. Campos EA, Reis JG. Representações sobre o uso do álcool por mulheres em tratamento em um centro de referência da cidade de São Paulo - Brasil. Interface-Comunicação, Saúde, Educação. 2010;34:243-54.

3. Moraes M. O modelo de atenção integral à saúde para tratamento de problemas decorrentes do uso de álcool e outras drogas: percepções de usuários, acompanhantes e profissionais. Ciênc Saúde Coletiva. 2008;13:121-33.

4. Assis DFF, Castro NT. Alcoolismo Feminino: início do beber alcoólico e busca por tratamento. Textos \& Contextos. (Porto Alegre) 2010;9:358-70.

5. Mendes MC, Cunha JRF, Nogueira AA. A mulher e o uso de álcool. Rev Bras Ginecol Obstet. 2011;33:323-7.

6. Souza JG, Lima JMB, Santos RS. Alcoolismo feminino: subsídios para a prática profissional da enfermagem. Esc Anna Nery. 2008;12:622-9.

7. Bardin L. Análise de Conteúdo. Lisboa: Ed. 70; $1977.280 \mathrm{p}$.

8. Boynton MH, Arkes J, Hoyle RH. Brief report of a test of differential alcohol risk using sibling attributions of paternal alcoholism. J Stud Alcohol Drugs. 2011 Nov;72:1037-40.

9. Katz LY, Cox BJ, Clara IP, Oleski J, Sacevich T. Substance abuse versus dependence and the structure of common mental disorders. Compr Psychiatry. 2011 Nov-Dec;52:638-43.

10. World Health Organization. Global status report on alcohol and health. Geneva: WHO; 2011. 
11. Vargas D. Nursing students' attitudes towards alcohol, alcoholism and alcoholics: a study of a Brazilian sample. J Nurs Educ Pract. 2012 Feb;2:1-8.

12. Cortaza Ramírez L, Villar Luís MA. Surge en mi otra mujer: significados del consumo de alcohol en mujeres mexicanas. Esc Anna Nery. 2008;12:693-8.

13. Monteiro CFS, Dourado GOL, Graça Júnior CAG, Freire AKN. Relatos de mulheres em uso prejudicial de bebidas alcoólicas. Esc Anna Nery. 2011;15:567-72.

14. Kachani AT, Okuda LS, Barbosa ALR, Brasiliano S, HochgrafPB. Aleitamento Materno: quanto o álcool pode influenciar na saúde do bebê? Pediatria. (São Paulo) 2008;30:249-56.

15. Del Ciampo LA, Ricco RG, Ferraz IS, Daneluzzi JC, Martinelli CE Junior. Prevalência de tabagismo e consumo de bebida alcoólica em mães de lactentes menores de seis meses de idade. Rev Paul Pediatr. 2009;27:361-5.

16. Kallen DW, Creuza RV, Marluce MS. Alcoolismo parental e fatores de risco associados. SMAD, Rev Eletrônica Saúde Mental Álcool Drog. (Ed. port.) 2011 set.-dez;7:161-7.

17. Chien YC, Huang YJ, Hsu CS, Chao JC, Liu JF. Maternal lactation characteristics after consumption of an alcoholic soup during postpartum 'doing-the-month' ritual. Public Health Nutr. 2009; 12:382-8.

18. Associação Brasileira de Estudos do Álcool e Outras Drogas. Álcool e Gravidez Síndro $\neg$ me Alcoólica Fetal, Tabaco e Outras Drogas [Internet]. 2008 [acesso $22 \mathrm{dez}$ 2012]. Disponível em: http://www.abead.com.br/midia/ exibMidia/?midia=2811. 